\title{
Jefferson
}

Thomas Jefferson University

The Medicine Forum

2016

\section{Anorectal Melanomas: Case Series}

Jinyu Zhang, MD

Thomas Jefferson University, Jinyu.Zhang@jefferson.edu

Matthew Sochat, MD

Temple University Hospital, Philadelphia PA

Edward R. Feller, MD

Warren Alpert Medical School of Brown University, Providence RI

Follow this and additional works at: https://jdc.jefferson.edu/tmf

Part of the Internal Medicine Commons, and the Oncology Commons

Let us know how access to this document benefits you

\section{Recommended Citation}

Zhang, MD, Jinyu; Sochat, MD, Matthew; and Feller, MD, Edward R. (2016) "Anorectal Melanomas: Case

Series," The Medicine Forum: Vol. 17, Article 9.

DOI: https://doi.org/10.29046/TMF.017.1.010

Available at: https://jdc.jefferson.edu/tmf/vol17/iss1/9

This Article is brought to you for free and open access by the Jefferson Digital Commons. The Jefferson Digital Commons is a service of Thomas Jefferson University's Center for Teaching and Learning (CTL). The Commons is a showcase for Jefferson books and journals, peer-reviewed scholarly publications, unique historical collections from the University archives, and teaching tools. The Jefferson Digital Commons allows researchers and interested readers anywhere in the world to learn about and keep up to date with Jefferson scholarship. This article has been accepted for inclusion in The Medicine Forum by an authorized administrator of the Jefferson Digital Commons. For more information, please contact: JeffersonDigitalCommons@jefferson.edu. 


\title{
Anorectal Melanomas: Case Series
}

\author{
Jinyu Zhang, MD1, Matthew Sochat, MD², and Edward R. Feller, MD³ \\ ${ }^{1}$ Thomas Jefferson University Hospital, Philadelphia, PA; ${ }^{2}$ Temple University Hospital, Philadelphia, PA; \\ 3The Warren Alpert Medical School of Brown University, Providence, RI
}

\section{INTRODUCTION}

Melanoma is the most common tumor to metastasize to the gastrointestinal (Gl) tract. $60 \%$ of patients who die with malignant melanoma have Gl involvement at autopsy. Symptomatic disease presenting during life is unusual. Anorectal melanoma, a rare tumor that represents $1 \%$ of all melanomas and less than $4 \%$ of all anal malignancies, manifests with a range of non-specific symptoms and physical exam findings ${ }^{2}$. In fact, about one-third of lesions may be amelanotic ${ }^{3,4}$. Initial misdiagnosis as a benign lesion such as hemorrhoid is common.

We report 3 cases of anorectal melanoma presenting as primary Gl complaints all without known cutaneous malignancy. Physicians must be aware of the clinical spectrum, anatomic distribution, and diagnostic difficulties associated with anorectal melanoma.

\section{CASE PRESENTATION}

\section{Case 1}

A 48 year-old woman presented with 1 week of bright red blood per rectum described as small amounts of blood surrounding otherwise brown stool. Physical exam was normal except for a firm mass on rectal exam. Hemoglobin was normal. Colonoscopy showed a 2-3 $\mathrm{cm}$ black mass inside the rectum at the dentate line. Histology was consistent with melanoma. A transanal excision documented a 4-5 mm thick melanoma invading the muscularis mucosa with vascular invasion. Surveillance chest radiograph 18 months later revealed lung metastasis. She had resection of the right lower lobe and right middle lobe. No cutaneous lesion was ever noted on her skin exam.

\section{Case 2}

An 84 year-old woman presented with several weeks of intermittent painless rectal bleeding. Initial rectal exam showed a hemorrhoid-like lesion. Hemoglobin was normal. She was treated symptomatically with hydrocortisone cream. Because her bleeding persisted after 3 weeks of treatment, further evaluation was performed. A non-pigmented tumor inside the rectum was discovered on retroflexion. Immunohistochemical staining of the lesion was strongly positive for S-100 and focally positive for HMB-45, both markers sensitive for melanocytic tumors. Transanal excision was performed. After a symptom-free interval of 2 years, intracerebral metastases were discovered. No cutaneous lesion was ever noted on skin exam.

\section{Case 3}

A 74 year-old woman presented with worsening fecal incontinence over several months complicated by sharp rectal pain, 25-pound weight loss, anorexia, constipation, tenesmus, and intermittent rectal bleeding. She described bleeding as flecks of blood on toilet tissue after defecation without gross blood in the stool. Family history was significant for head and neck cancer, breast cancer, and lung cancer. A non-pigmented $3 \mathrm{~cm}$ anorectal mass was found at colonoscopy. Biopsy was positive for melanoma. She underwent an abdominoperineal resection with colostomy. Distant spread of tumor was not identified in the initial post-op evaluation; skin exam was negative for melanoma both prior to and at the time of diagnosis.

\section{DISCUSSION}

Compared to cutaneous melanoma, non-cutaneous melanoma is rare and located in ocular sites or sites such as anorectal, vaginal, and nasal mucosa. The anorectum is the third most frequent mucosal site for melanoma, after head and neck and the female genital tract. Mucosal melanoma occurs predominantly in older individuals; $49 \%$ present at age 70 or older ${ }^{5}$. A recent report, however, by Ellis and co-workers described cases of an 11-year-old and a 19-year-old patient with anorectal melanoma .

Race, ethnicity, and gender differences exist. In the U.S., fewer than $3 \%$ of cutaneous melanomas are diagnosed in African Americans and Hispanics; however, these minority groups represent approximately $9 \%$ of individuals diagnosed with mucosal melanoma ${ }^{5}$. Women are more likely than men to be diagnosed with anorectal melanoma (male: female ratio 3:4). Sun exposure is not a risk factor for mucosal melanoma². Collected data suggest an increased risk in HIV-positive patients?

Presenting complaints are non-specific and mimic common non-neoplastic disorders such as hemorrhoids. As described in each of our cases, rectal bleeding was the most frequent complaint followed by mass sensation and tenesmus. Other symptoms include anal pruritus, changes in bowel habits, and rectal pain or prolapse. Prior to histology, other possible malignant diagnoses include epidermoid carcinoma, basal cell carcinoma, adenocarcinoma, and more unusual tumors - anaplastic sarcoma, lymphoma, and undifferentiated cylindrical cellular carcinoma. The macroscopic appearance of anorectal melanoma ranges from a polypoid mass to a pigmented or non-pigmented ulceration 4.8 . 


\section{The Medicine Forum, Vol. 17 [2016], Art. 9}

Diagnosis is often delayed because most symptoms are non- specific or thorough anorectal examinations, including anoscopy, are not performed. Identifiable lesions are pigmented in about two-thirds of cases ${ }^{3,4}$. Commonly, as in each of our three cases, no skin lesions are noted. One case series suggests that a benign anorectal condition was the initial diagnosis in 46 of 79 patients ${ }^{9}$. Cases misdiagnosed as hemorrhoids had a statistically significant decrease in survival with the average duration of symptoms before diagnosis being between 4 and 6 months. In a review of mucosal melanoma, one study found that anorectal melanomas had been misdiagnosed in about two thirds of patients - most commonly as hemorrhoids, adenocarcinoma, or polyp ${ }^{10}$.

Terminology varies depending on which system is used. One popular classification uses the following criteria: melanoma is termed by location - anal if below dentate line, rectal if above, and anorectal if around. No one schema is accepted or used universally. Purely rectal melanoma generally represent less than $10 \%$ of cases $^{11}$.

Diagnosing anorectal melanoma is challenging. Complete skin examination and review of previous skin biopsies are important ${ }^{12}$. Physical examination must include visual inspection of the anus, digital examination, and anoscopy and/or proctoscopy (retroflexion of endoscope in the rectum looking for evidence of thrombosis or related pathology 12,13

Anorectal melanoma is highly lethal. In many patients, this malignancy presents as localized, resectable disease; however, initially undetected metastases are common; thus, long-term survival is unusual. As many as a third of patients have metastatic disease at presentation and more than $80 \%$ of patients die of distant metastatic disease within 5 years $^{15}$. Location also plays a role in survival. A small retrospective study using the classification described above found that two-thirds of rectal and anorectal melanomas recurred systemically whereas anal melanoma tended to recur first within sentinel lymph nodes ${ }^{16}$.

\section{KEY POINTS}

Any patient with a history of melanoma presenting with digestive symptoms, especially anorectal, should have a careful diagnostic evaluation. This malignancy can masquerade as refractory hemorrhoid disease as in our second case. Diagnosis of melanoma should be considered in hemorrhoids that enlarge; are pigmented, ulcerated or indurated; and persist despite conservative therapy. Anorectal melanoma may present (as in our third case) as a non-pigmented mass, and be associated with small amounts of rectal bleeding, pain, itching, or incontinence. Bowel complaints of any magnitude in adults with a known melanoma should prompt consideration of metastatic Gl involvement in patients with a known melanoma. Immunohistochemical staining is useful in diagnosing a questionable lesion. Despite its rarity, due to its significant mortality, anorectal melanoma should remain on the differential in unusual anorectal lesions.

\section{REFERENCES}

1. M. Lens, V. Bataille, and Z. Krivokapic. Melanoma of the small intestine. Lancet Oncology 2009; 10(5): 516-521.

2. D. Row and M. R. Weiser MR. Anorectal melanoma. Clinics in Colon and Rectal Surgery 2009; 22(2): 120-126.

3. A. Hillenbrand, T. F. Barth, D. Henne-Bruns, and A. Formentini. Anorectal amelanotic melanoma. Colorectal Disease: The Official Journal of the Association of Coloproctology of Great Britain and Ireland 2008; 10(6): 612-615.

4. L. M. Schuchter, R. Green, and D. Fraker. Primary and metastatic diseases in malignant melanoma of the gastrointestinal tract. Current Opinion in Oncology 2000; 12(2): 181-185.

5. A. E. Chang, L. H. Karnell, and H. R. Menck. The National Cancer Data Base report on cutaneous and noncutaneous melanoma: a summary of 84,836 cases from the past decade. The American College of Surgeons Commission on Cancer and the American Cancer Society. Cancer 1998; 83(8): 1664-1678.

6. Z. M. Ellis, A. D. Jassim, and M. R. Wick. Anorectal melanoma in childhood and adolescence. Annals of Diagnostic Pathology 2010; 14(2): 69-73.

7. A. Burgi, S. Brodine, S. Wegner et al. Incidence and risk factors for the occurrence of non-AIDS-defining cancers among human immunodeficiency virus-infected individuals. Cancer 2005; 104(7): 1505-1511.

8. D. J. Chute, J. B. Cousar, and S. E. Mills. Anorectal malignant melanoma morphologic and immunohistochemical features. American Journal of Clinical Pathology 2006; 126(1): 93-100.

9. S. Zhang, F. Gao, and D. Wan. Effect of misdiagnosis on the prognosis of anorectal malignant melanoma. Journal of Cancer Research and Clinical Oncology 2010; 136(9): 1401-1405.

10. M. Mihajlovic, S. Vlajkovic, P. Jovanovic, and V. Stefanovic. Primary mucosal melanomas: a comprehensive review. International Journal of Clinical and Experimental Pathology 2012; 5(8): 739-753.

11. L. B. Yap and P. Neary. A comparison of wide local excision with abdominoperineal resection in anorectal melanoma. Melanoma Research 2004; 14(2): $147-150$.

12. J. N. Cormier, Y. Xing, L. Feng et al. Metastatic melanoma to lymph nodes in patients with unknown primary sites. Cancer 2006; 106(9): 2012-2020.

13. D. E. Rivadeneira, S. R. Steele, C. Ternent et al. Practice parameters for the management of hemorrhoids (revised 2010). Diseases of the Colon and Rectum 2011; 54(9): 1059-1064.

14. C. Thibault, P. Sagar, S. Nivatvongs et al. Anorectal melanoma--an incurable disease? Diseases of the Colon and Rectum 1997; 40(6): 661-668. 